\title{
The Land Equivalent Ratio and Its Mutualistic Implications: Computational Approach
}

\author{
${ }^{1}$ E.N. Ekaka-A, ${ }^{2}$ N.E.S. Lale, ${ }^{3}$ C.C. Wokocha, \\ ${ }^{I}$ Department of Mathematics and Computer Science, Rivers State University of Science And \\ Technology, Port Harcourt, Nigeria, \\ ${ }^{2,3}$ Department of Crop And Soil Science, Faculty Of Agriculture, University Of Port Harcourt, Port \\ Harcourt, Nigeria, And
}

\begin{abstract}
In this paper, we develop the theoretical and computational per-spectives of tackling and calculating the concept of land equivalent ratio (LER). We apply this idea to discuss its application in a mutualistic interaction.

Criteria for evaluating different intercropping situations are suggested also in this paper, and the land equivalent ration (LER) concept is considered for situations where intercropping has to be compared with growing each crop in pure stand. The need to use different standardizing sole crop yields in forming LERs is discussed, and a method of calculating an 'effective LER' is proposed to evaluate situations where the yield proportions achieved in intercropping are different from those that might be required by a farmer. The possible importance of effective LERs in indicating the proportions of crops likely to give biggest yield advantages is discussed.
\end{abstract}

Keywords: and phrases. LER, Mutualism, Numerical Simulation, ODE45, ODE23.

\section{Introduction}

What is known about the concept of the Land Equivalent Ratio? Within the research activities of crop science and crop protection, the benefits of growing two or more crops together or intercropping are enormous. Assessing these benefits invloves measuring productivity by using the concept of the Land Equivalent Ratio (LER) ([4], [5]). One of the means of understanding the dynamics of the concepts of biological interactions is through the process of mathematical modelling ([2], [3]).

Following our previous mathematical modelling of Lotka-Volterra-like competi- tion models ([2], [3]), we have selected the following parameters in the analysis of the problem we propose to study in this paper: the intraspecific coefficient values of 0.00165 ; the interspecific coefficient values of 0.0016 for the first variety of sorghum and 0.0015 for the second variety of sorghum; the daily intrinsic growth rate of

0.15 for the second variety of sorghum and the daily intrinsic growth rate of 0.16 for the first variety of sorghum. Without a detailed mathematical analysis that leads to the next vital background ecological information, using this set of model parameters provides us with four possible steady-state solutions namely the trivial steady-state $(0,0)$ where the two varieties of sorghum will go extinct followed by two other steady-state solutions $(0,90.49)$ and $(96.52,0)$ when either of the varieties will tend to survive at its carrying capacity. These two varieties of sorghum will coexist when the biomass of the first variety is 72.52 and the biomass of the second variety is 24.86 . Since the inhibiting effect of the second variety on the growth of variety 1 is 0.96 [ obtained by dividing the interspefic coefficient of 0.0016 of the first variety by its intraspecific coefficient of 0.00165] ([2]) is less than the ratio of the carrying capacity of the first variety to the carrying capacity of the second variety [ that is dividing 96.52 by 90.49], it follows that the first variety of sorghum will survive under this simplifying tested formula. The second variety of sorghum will also survive because the inhibiting effect of the first variety on the growth of the second variety of sorghum is 0.9049 which is less than 0.9375 being the ratio of the carrying capacity of the second variety of sorghum to the carrying capacity of the first variety of sorghum.

This paper is organized into the following sections. Section 1 is focused in defin- ing the concept of the Land Equivalent Ratio and its practical application to agri- cultural data. Section 2 will discuss LER as it affects the biological interaction of mutualism ([3]). These calculations will be quantitatively discussed and analysed. 


\section{Numerical Calculation of the LER: Application to Mutualistic}

\section{Interaction}

In this section, we are interested in studying the effect of varying the daily intrinsic growth rate of a variety of sorghum on our estimated value of the Land Equivalent Ratio (LER). The goal is to investigate how this growth rate variation affects the incidence of each type of interaction following our recent mathematical modelling idea in the context of plant species interactions in a harsh environment ([3]).

By the assumption of the logistic growth of sorghum as a crop and invoking how the LER is calculated, we will set out to illustrate how we have carried out a systematic numerical calculation of the LER by using a powerful written MATLAB program which is based on the ODE45 simulation technique.

2.1. When the daily intrinsic growth rates are 0.04 and 0.06 . If the daily intrinsic growth rates are 0.04 grams per day and 0.06 grams per day and using a complex interspecific competition model, we have used our MATLAB program to calculate the LERs for the first variety of sorghum hereby called species $\mathrm{N}_{1}$ and also for the second variety of sorghum called species $\mathrm{N}_{2}$. The assumption is that these two varieties of sorghum are interacting and competing for a limited resource within the Northern Nigeria agricultural setting. Following [4] and [5], to calculate the partial LER of $\mathrm{N}_{1}$ we have divided its intercrop yield within an appropriate unit by the value of its pure stand or monoculture yield. On the basis of the popular Lotka-Volterra model formulation or educational model in mathematical biology and our MATLAB simulation program, the following partial LER and total LER are calculated. These calculations are considered for a growing season of 90 days using our ODE45 MATLAB program. The unit of growth is grams.

\begin{tabular}{|l|l|l|l|l|l|l|l|l|}
\hline Weekly Data & \multicolumn{9}{l}{ Sorghum Growth Data } \\
\hline week number & $\mathrm{S}_{1}$ & $\mathrm{~S}_{1 \mathbf{i}}$ & $\mathrm{S}_{2}$ & $\mathrm{~S}_{2} \mathbf{i}$ & $\mathrm{S}_{1}$ & $\mathrm{~S}_{1} \mathbf{i}$ & $\mathrm{S}_{2}$ & $\mathrm{~S}_{2} \mathbf{i}$ \\
\hline 1 & 1.00 & 1.00 & 2.00 & 2.00 & 1.00 & 1.00 & 2.00 & 2.00 \\
\hline 2 & 1.35 & 2.58 & 5.55 & 5.49 & 1.56 & 2.58 & 5.56 & 5.49 \\
\hline 3 & 1.96 & 6.45 & 14.48 & 14.11 & 2.58 & 6.45 & 14.55 & 14.11 \\
\hline 4 & 3.24 & 15.06 & 32.65 & 31.31 & 4.86 & 15.06 & 33.08 & 31.31 \\
\hline 5 & 6.74 & 30.60 & 58.02 & 54.56 & 11.37 & 30.60 & 59.86 & 54.56 \\
\hline 7 & 17.24 & 50.40 & 82.19 & 73.73 & 31.60 & 50.40 & 88.83 & 73.73 \\
\hline 8 & 46.35 & 66.86 & 107.46 & 84.07 & 84.52 & 66.86 & 129.86 & 84.07 \\
\hline 9 & 107.93 & 76.35 & 152.34 & 88.40 & 182.76 & 76.35 & 210.80 & 88.40 \\
\hline 10 & 204.22 & 80.70 & 236.66 & 90.00 & 326.46 & 80.70 & 345.45 & 90.00 \\
\hline 11 & 333.46 & 82.50 & 359.18 & 90.60 & 501.63 & 82.50 & 514.43 & 90.60 \\
\hline 12 & 479.85 & 83.20 & 499.90 & 90.80 & 668.33 & 83.20 & 676.52 & 90.80 \\
\hline 13 & 614.12 & 83.47 & 629.33 & 90.87 & 793.40 & 83.47 & 797.06 & 90.87 \\
\hline
\end{tabular}

Table 1. Calculation of Total LER; length of the growing season is 90 days; growth data of sorghum based on a 90 day growing sea- son; growth data $\mathrm{S}_{1}$ represent the growth data for the first variety of sorghum in competition with sorghum variety $\mathrm{S}_{2}$; growth data $\mathrm{S}_{1} \mathbf{i}$ represent the growth data for variety $\mathrm{S}_{1}$ growing in isolation of variety $\mathrm{S}_{1}$; growth data $\mathrm{S}_{2}$ represent the growth data for the sec- ond variety of sorghum in competition with sorghum variety $\mathrm{S}_{1}$; growth data $\mathrm{S}_{2} \mathbf{i}$ represent the growth data for variety $\mathrm{S}_{2}$ growing in isolation of variety $\mathrm{S}_{2}$

What do we learn from this Table 1? At the end of the 90 days when the intrinsic growth rate is 0.04 , our partial LER for $\mathrm{S}_{1}$ is 8.5537 (this estimated value was obtained by dividing 714.85 by 83.57 which are the growth data at the end of 90 days for variety $\mathrm{S}_{1}$ ) which is followed by the partial LER for S2 having an estimated value of 7.9986 (this estimated value was obtained by dividing 727.04 by 90.90 which are the growth data at the end of 90 days for variety S2). By adding the values of these partial LERs for varieties $S_{1}$ and $S_{2}$, we obtained our estimated total LER of 16.5523. This value of total LER shows the positive impact of growing sorghum variety $S_{1}$ with variety $S_{2}$. Similarly, when the value of the daily intrinsic growth rate is 0.06 , the total LER is 20.01 which indicates a positive impact of growing sorghum varieties together.

However, the interacting behaviour is called mutualistic interaction because the species growing together grow better than the isolated varieties.

By considering other variations of the daily intrinsic growth rate, we are inter- ested in finding 
how the qualitative behaviour of our LER is responding and which type of biological interaction is dominant.

In order to validate the appropriateness of using the notion of LER for the two varieties of sorghum, it would be useful to measure the size of the error between the LER of S1 and the LER of S2 when the daily intrinsic growth rate is 0.04 . In this scenario, our estimated values of the p-norms of the difference between the LERs are 5.97 for the 1-norm, 1.94 for the 2-norm and 0.84 for the $\infty$-norm. In contrast, when the daily intrinsic growth rate is 0.06 , our estimated values of the p-norms of the difference between the LERs are 6.12 for the 1-norm, 1.95 for the 2-norm and

0.83 for the $\infty$-norm.

2.2. When the daily intrinsic growth rates are 0.08 and 0.1 . In this sub- section, we will consider the situation when the estimated values of our intrinsic growth rates are 0.08 and 0.1 . Here, we will present a similar set of partial and total LER numerical calculations. Without delving into detailed explanation as we have done above, our next set of results are presented below.

\begin{tabular}{|c|c|c|c|c|c|c|c|c|}
\hline \multirow{2}{*}{\begin{tabular}{|l} 
Weekly Data \\
week number
\end{tabular}} & \multicolumn{8}{|c|}{ Sorghum Growth Data } \\
\hline & $\mathrm{S}_{1}$ & $\mathrm{~S}_{1 \mathbf{i}}$ & $\mathrm{S}_{2}$ & $\mathrm{~S}_{2 \mathbf{i}}$ & $\mathrm{S}_{1}$ & $\mathrm{~S}_{1 \mathbf{i}}$ & $\mathrm{S}_{2}$ & $\mathrm{~S}_{2 \mathbf{i}}$ \\
\hline 1 & 1.00 & 1.00 & 2.00 & 2.00 & 1.00 & 1.00 & 2.00 & 2.00 \\
\hline 2 & 1.80 & 2.58 & 5.60 & 5.49 & 2.10 & 2.58 & 5.60 & 5.49 \\
\hline 3 & 3.40 & 6.45 & 14.60 & 14.11 & 4.40 & 6.45 & 14.70 & 14.11 \\
\hline 4 & 7.30 & 15.06 & 33.70 & 31.31 & 10.70 & 15.06 & 34.50 & 31.31 \\
\hline 5 & 18.80 & 30.60 & 62.70 & 54.56 & 30.40 & 30.60 & 67.20 & 54.56 \\
\hline 6 & 54.90 & 50.40 & 100.30 & 73.73 & 89.20 & 50.40 & 119.40 & 73.73 \\
\hline 7 & 140.10 & 66.86 & 167.80 & 84.07 & 214.00 & 66.86 & 227.00 & 84.07 \\
\hline 8 & 283.70 & 76.35 & 298.90 & 88.40 & 414.50 & 76.35 & 418.30 & 88.40 \\
\hline 9 & 481.40 & 80.70 & 489.20 & 90.00 & 662.40 & 80.70 & 658.20 & 90.00 \\
\hline 10 & 689.50 & 82.50 & 690.40 & 90.60 & 878.00 & 82.50 & 867.60 & 90.60 \\
\hline 11 & 850.90 & 83.20 & 848.00 & 90.80 & 1015.40 & 83.20 & 999.80 & 90.80 \\
\hline 12 & 950.70 & 83.47 & 943.30 & 90.87 & 1085.70 & 83.47 & 1067.70 & 90.87 \\
\hline 13 & 1002.50 & 83.57 & 993.40 & 90.90 & 1117.60 & 83.57 & 1099.00 & 90.90 \\
\hline
\end{tabular}

Table 2. Calculation of Total LER; length of the growing season is 90 days; growth data of sorghum based on a 90 day growing sea- son; growth data $\mathrm{S}_{1}$ represent the growth data for the first variety of sorghum in competition with sorghum variety $S_{2}$; growth data $S_{1} \mathbf{i}$ represent the growth data for variety $S_{1}$ growing in isolation of variety $\mathrm{S}_{1}$; growth data $\mathrm{S}_{2}$ represent the growth data for the sec- ond variety of sorghum in competition with sorghum variety $\mathrm{S}_{1}$; growth data $\mathrm{S}_{2} \mathbf{i}$ represent the growth data for variety $\mathrm{S}_{2}$ growing in isolation of variety $\mathrm{S}_{2}$

What do we learn from this Table 2? At the end of the 90 days when the intrinsic growth rate is 0.08 , our partial LER for $\mathrm{S}_{1}$ is 11.9954 whereas our partial LER for $\mathrm{S}_{2}$ is 10.9296 . By adding the values of these partial LERs for varieties $S_{1}$ and $S_{2}$, we obtained our estimated total LER of 22.925. This value of total LER shows the positive impact of growing sorghum variety $\mathrm{S}_{1}$ with variety $\mathrm{S}_{2}$. Similarly, when the value of the daily intrinsic growth rate is 0.1 , the total LER is 25.4638 which indicates a positive impact of growing sorghum varieties together.

We will aim at measuring the size of the error between the LER of $\mathrm{S}_{1}$ and the LER of $\mathrm{S}_{2}$ when the daily intrinsic growth rates are 0.08 and 0.1 . When the daily intrinsic growth rate is 0.08 , our estimated values of the p-norms of the difference between the LERs are 6.89 for the 1-norm, 2.23 for the 2-norm and 1.07 for the $\infty$-norm. In contrast, when the daily intrinsic growth rate is 0.1 , our estimated values of the p-norms of the difference between the LERs are 8.24 for the 1-norm,

2.78 for the 2 -norm and 1.28 for the $\infty$-norm.

Henceforth, we will only present our other key results for other variations of the daily intrinsic growth rate for the first plant species. These results would concern the total LER for the two varieties of sorghum under a mutualistic interaction, the size of the error between the the LER of $\mathrm{S}_{1}$ and the LER of $S_{2}$ when the daily intrinsic growth rate ranges from the value of 0.105 to 0.18 and the limiting biomasses between two interacting sorghum varieties and their isolated components. Our estimated calculations are presented as 
The Land Equivalent Ratio and Its Mutualistic Implications: Computational Approach

\begin{tabular}{|c|c|c|c|c|c|c|c|c|c|c|}
\hline \multicolumn{11}{|c|}{ Paramete Data } \\
\hline value & LERS $_{1}$ & LERS $_{2}$ & TLER & p1n & $\mathrm{p} 2 \mathrm{n}$ & pmaxn & $\mathrm{S}_{1}$ & $S_{1 i}$ & $\mathrm{~S}_{2}$ & $\mathrm{~S}_{2 \mathbf{i}}$ \\
\hline 0.105 & 13.6976 & 12.3604 & 26.0580 & 8.702 & 2.960 & 1.337 & 1144.7 & 83.57 & 1123.5 & 90.90 \\
\hline 0.110 & 14.0141 & 12.6259 & 26.6400 & 9.148 & 3.155 & 1.388 & 1171.2 & 83.57 & 1147.6 & 90.90 \\
\hline 0.115 & 14.3343 & 12.8785 & 27.2128 & 9.70 & 3.36 & 1.46 & 1197.9 & 83.57 & 1170.60 & 90.90 \\
\hline 0.120 & 14.6304 & 13.1438 & 27.7740 & 10.35 & 3.57 & 1.49 & 1222.7 & 83.57 & 1194.70 & 90.90 \\
\hline 0.125 & 14.9351 & 13.3932 & 28.3283 & 10.99 & 3.80 & 1.54 & 1248.2 & 83.57 & 1217.40 & 90.90 \\
\hline 0.130 & 15.2337 & 13.6426 & 28.8763 & 11.77 & 4.02 & 1.59 & 1273 & 83.57 & 1240 & 90.90 \\
\hline 0.135 & 15.5316 & 13.8877 & 29.4193 & 12.78 & 4.30 & 1.64 & 1298 & 83.57 & 1262.30 & 90.90 \\
\hline 0.140 & 15.8141 & 14.1420 & 29.9561 & 13.85 & 4.55 & 1.69 & 1321.60 & 83.57 & 1285.40 & 90.90 \\
\hline 0.145 & 16.1212 & 14.3708 & 30.4920 & 15.00 & 4.85 & 1.75 & 1347.30 & 83.57 & 1306.20 & 90.90 \\
\hline 0.150 & 16.4144 & 14.6086 & 31.023 & 16.195 & 5.158 & 1.834 & 1371.80 & 83.57 & 1327.90 & 90.90 \\
\hline 0.155 & 16.6871 & 14.8608 & 31.5479 & 17.36 & 5.46 & 1.940 & 1394.6 & 83.57 & 1350.8 & 90.90 \\
\hline 0.160 & 16.9769 & 15.0963 & 32.0732 & 18.60 & 5.80 & 2.06 & 1418.8 & 83.57 & 1372.20 & 90.90 \\
\hline 0.165 & 17.2571 & 15.3361 & 32.5952 & 19.83 & 6.13 & 2.18 & 1442.40 & 83.57 & 1394.00 & 90.90 \\
\hline 0.170 & 17.5491 & 15.5675 & 33.1166 & 21.07 & 6.47 & 2.34 & 1466.60 & 83.57 & 1415.00 & 90.90 \\
\hline 0.175 & 17.8319 & 15.8042 & 33.6361 & 22.4307 & 6.85 & 2.50 & 1490.20 & 83.57 & 1436.50 & 90.90 \\
\hline 0.180 & 18.1131 & 16.0411 & 34.1542 & 23.80 & 7.24 & 2.68 & $\mid 1513.70$ & 83.57 & 1458.00 & 90.90 \\
\hline
\end{tabular}

Table 3. Calculation of Total LER; length of the growing season is 90 days; growth data of sorghum based on a 90 day growing season; $S_{1}$ represents the limiting biomass for the first variety of sorghum in competition with sorghum variety $\mathrm{S}_{2} ; \mathrm{S}_{1} \mathbf{i}$ represents the limiting biomass for the variety $\mathrm{S}_{1}$ growing in

isolation of va- riety $\mathrm{S}_{1} ; \mathrm{S}_{2}$ represents the limiting biomass for the second variety of sorghum in competition with sorghum variety $\mathrm{S}_{1} ; \mathrm{S}_{2} \mathbf{i}$ represents the limiting biomass for the variety $\mathrm{S}_{2}$ growing in isolation of va- riety $\mathrm{S}_{2}$

Here, the notations $p 1 n$ represents $p-1$ norm, $p 2 n$ represents $p-2$ norm and pmaxn represents the $\mathrm{p}$ maximum norm or the infinity norm.

\section{Discussion of Results}

We observe that as the daily intrinsic growth rate for species $S_{1}$ is slightly in- creased, the total LER and the size of the error between the LERs similarly in- crease. Within this interval space of increase in the value of the intrinsic growth rate, our numerical calculation estimates clearly show that the factor of mutualism is a dominant type of species interaction which we have not seen elsewhere.

\section{Concluding Remarks and Further Research}

In this study, we have developed the theoretical and analytical perspectives of the concept of land equivalent ratio (LER) in which this idea has been systematically applied to demonstrate that mutualism is a dominant type of species interaction. The implication of this key result is that when two crop species are interacting mutualistically for a limited resource in the environment, the consistent positive value of the total LER clearly shows the positive advantage of growing one crop species with another crop species and that more land is required to sustain this beneficial interaction behaviour.

This present result is an extension of our first earlier study. Our numerical approach to tackling similar crop data is proposed to relate the idea of the (LER) to other types of species interactions such as commensalism and predation.

\section{References}

[1] R.B. Banks, Growth and Diffusion Phenomena Mathematical Frameworks and Applications, Springer-Verlag, 1994.

[2] E.N. Ekaka-a, Computational and Mathematical Modelling of Plant Species Interactions in a Harsh Climate, PhD Thesis, Department of Mathematics, The University of Liverpool and The University of Chester, United Kingdom, 2009.

[3] N.J. Ford, P.M. Lumb, E. Ekaka-a, Mathematical modelling of plant species interactions in a harsh climate, Journal of Computational and Applied Mathematics 234, (2010), 2732-2744.

[4] S. Giliessman, Agroecology: Ecological Processes in Sustainable Agriculture, Sleeping Bear Press, MI, 1998.

[5] P. Sullivan, Intercropping Principles and Production Practices, Appropriate Technology Trans- fer for Rural Areas (ATTRA), Fayetteville, AR, 1998. 